\title{
A contribution to algebraic approach for a CAD/CAPP specification
}

\author{
João Almeida das Rosas, Goran D. Putnik \\ University of Minho, Production Systems Engineering Centre \\ Azurem, 4800 Guimaraes, Portugal, fax.: +351-53-510268 \\ e-mail: \{mpic_jar, putnikgd\}@eng.uminho.pt
}

\begin{abstract}
Algebraic specification of processes is an emerging research issue in last years as a tool for formalization of system design processes, essentially in computer sciences. By a formal specification we are able to prove formally rigirousness of functional requirements and theirs implementations. Traditional approach to system implementation based on trial-and-error (i.e. experimentation) practise is informal and inefficient for a large number of systems. The paper contributes to application of a formal algebraic specification, as a rigorous formalism, in manufacturing systems design and control, specifically for the CAD/CAPP systems specification. The formal algebraic specifiication provides a rigorous base for its correct specification and implementation in a programming language, i.e. for efficient and correct development of corresponded software tools.
\end{abstract}

Keywords

Algebra for Manufacturing Systems, Universal Algebra, Formal Theory for Manufacturing Systems, CAD/CAPP, CAD/CAPP Algebraic Model

\section{INTRODUCTION - FROM SPECIFICATION TO IMPLEMENTATION}

Automation and integration of manufacturing system functions, for example the CAD/CAPP (Computer Aided Design/Computer Aided Process Planning) function, pass through their specification in the first phase and implementation in the second phase. As a relation between specification and implementation is one-to-many, there is a certain risk to introduce redundancies in specification from the reason of details of implementation or in order to achieve some performances. But, from the other side, gain on performances means loosing the clarity of the

\footnotetext{
The original version of this chapter was revised: The copyright line was incorrect. This has been corrected. The Erratum to this chapter is available at DOI: 10.1007/978-0-387-35390-6_58 
system representation. Thus, it is necessary to implement the system from system specification which is as much abstract as possible, capturing only the essence of the system, maintaining the representation clarity and facilitating the system implementation. This necessary abstraction in the system specification requires a formal specification of the system through rigorous mathematical language. An algebraic approach is certainly one rigorous formal base for achieving it.

In the paper we introduce basic definitions from the abstract/universal algebra, together with issues on implementation in computer environment, followed by one simple example representing a framework for $\mathrm{CAD} / \mathrm{CAPP}$ algebraic specification.

\section{ALGEBRAIC SPECIFICATION - BASIC DEFINITIONS}

\subsection{Abstract syntax}

Definition 2.1 (Signature) A signature $\sum$ is a set of mappings $\Sigma: \Omega \rightarrow \mathrm{S}^{*} \mathrm{xS}$, where $\mathrm{S}$ is a finite nonempty set of sorts (of objects) symbols and $\Omega$ is a finite nonempty set of operation symbols.

For all $\sigma \in \Omega$, and $\mathrm{n} \geq 1$ if $\left.\Sigma(\sigma)=\left(<\mathrm{s}_{1}, \ldots, \mathbf{s}_{\mathrm{n}}\right\rangle, \mathrm{s}\right)$ we write, also, $\sigma: \mathbf{s}_{1} \times \ldots \times \mathbf{s}_{\mathrm{n}} \rightarrow \mathbf{s}$.

Definition 2.2 (Constants) The set of constants $C_{s}$ of the sort $\mathrm{s} \in \mathrm{S}$ in a signature $\sum$ is given by:

$$
C_{S} \stackrel{\text { def }}{=}\left\{\sigma \in \Omega \mid \sum(\sigma)=(<>, s)\right\}
$$

We write, also, $\sigma:<>\rightarrow \mathrm{s}$, or, simply, $\sigma: \rightarrow \mathrm{s}$. Constants are symbols of special operations with 0 -arity. In other words, constants are algebraic values. They allow a syntactic construction of terms by the symbols from the signature.

Definition 2.3 (Homogeneous/Heterogeneous Signatures) A signature $\Sigma: \Omega \rightarrow$ $S^{*} x S$, is homogeneous if $|S|=1$ and heterogeneous if $|S|>1$.

In real situations signatures are, usually, heterogeneous.

Definition 2.4 ( $\Sigma$-term) Let $\Sigma: \Omega \rightarrow S^{*} x S$ be a signature. For all sort $s \in S$ it is possible to construct the set of all " $\sum$-terms" of the sort $s$, which is the minimum set satisfying the following clauses:

1. all constants $\sigma: \rightarrow \mathrm{s}$ are $\sum$-terms of the sorts $\mathrm{s}$;

2. for all operations $\sigma: s_{1} \times \ldots \times s_{n} \rightarrow s$, if $t_{i}, 1 \leq i \leq n$, is a $\sum$-term of the sort $\mathrm{s}_{\mathrm{i}}$, then $\sigma\left(\mathrm{t}_{1}, \ldots, \mathrm{t}_{\mathrm{i}}, \ldots, \mathrm{t}_{\mathrm{n}}\right)$ is a $\sum$-term of the sort $\mathrm{s}$.

The set of all $\sum$-terms is denoted $\mathrm{W}_{\Sigma, \mathrm{s}} . \mathrm{A} \sum$-term is called a $\sum$-word (phrase).

\subsection{Concrete syntax}

Definition of a concrete syntax means to construct a grammar from a signature, transforming operation and sort symbols from an abstract specification to a more concrete one. 
Let $\mathrm{G}=<\mathrm{N}, \mathrm{T}, \mathrm{S}, \mathrm{P}>$ be a Context-Free Grammar $(\mathrm{CF})$, where $\mathrm{N}$ is a finite set of non-terminal symbols, $\mathrm{T}$ is a finite set of terminal symbols, $(\mathrm{N} \cap \mathrm{T}) \neq \varnothing, \mathrm{S} \in \mathrm{N}$ is the initial (sentence) symbol and $\mathrm{P} \subseteq \mathrm{Nx}(\mathrm{N} \cup T)^{*}$ is the set of productions of G. Definition of a $\mathrm{CF}$ grammar $^{1}$, among $\mathrm{n}$ possible, is as follows:

1. Each sort symbol $\mathrm{s} \in \mathrm{S}$ is a non-terminal symbol of a grammar;

2. Each operation symbol $\sigma \in \Omega$ is a production of a grammar;

3. In a set of production introduce necessary terminal symbols, making a grammar recognisable by an automata.

\subsection{Semantics}

A phrase semantics generated by the corresponded signature is introduced.

Definition 2.5 ( $\Sigma$-algebra) Let $\Sigma: \Omega \rightarrow \mathrm{S}^{*} \mathrm{xS}$ be a signature. The pair $A=\left(A_{S}\right.$, $\left.A_{\Omega}\right)$ is a $\sum$-algebra if: $A_{S}$ is a finite set of $\operatorname{Sets}^{2} ; A_{\Omega}$ is a finite set of functions of sets to sets (of Sets), such that, for each $\sigma \in \Omega$ for which $\sum(\sigma)=\left(\left\langle\mathbf{s}_{1}, \ldots, \mathbf{s}_{\mathrm{n}}\right\rangle, \mathbf{s}\right)$, the function $A_{\Omega}(\sigma)$, on the corresponded sets of Sets, respects the functionality $\sigma$, in accordance with a diagram:

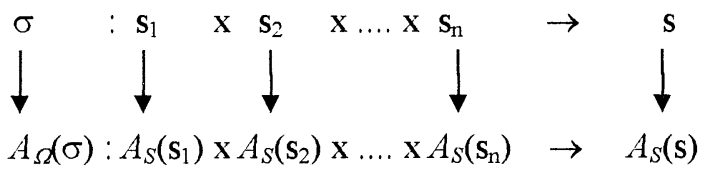

The sets $A_{S}, A_{\Omega}$ are called the carrier sets. A $\sum$-algebra could be viewed as a set with a structure. It consists of a set $A$ and an interpretation over $A$ of each symbol of the signature $\Sigma$. We say that a $\sum$-algebra gives a semantics to a signature, that is, a correspondence between each $\sigma \in \Omega$ and $\mathrm{s} \in \mathrm{S}$, and corresponded semantics domains. The semantics domain could be mathematics (for example: set theory, ...), programming languages (for example: C, Pascal, Lisp, ...), etc. Normally it is applied a mathematical specification of the semantics, being an application generated automatically by some software tool (for example: Vmd, Camila).

Whenever we define a $\sum$-algebra we define a model of the signature.

Definition 2.6 (Initial Model) Let $\Sigma: \Omega \rightarrow \mathrm{S}^{*} \mathrm{xS}$ be a signature. Let $\mathrm{W}$ be a $\Sigma$ algebra such that $\mathrm{W}_{\mathrm{S}}(\mathrm{s})=\mathrm{W}_{\Sigma, \mathrm{s}}$ for every $\mathrm{s} \in \mathrm{S}$ and for every $\sigma \in \Omega$ such that $\sigma$ : $\mathrm{s}_{1} \times \ldots \times \mathrm{s}_{\mathrm{n}} \rightarrow \mathrm{s}$

$$
\begin{gathered}
\mathrm{W}_{\Omega}(\sigma) \quad: \quad \mathrm{W}_{\sum, s 1} \times \ldots \mathrm{W}_{\Sigma, \mathrm{sn}} \\
\left\langle\mathrm{t}_{1}, \ldots, \mathrm{t}_{\mathrm{n}}\right\rangle \stackrel{\mathrm{W}}{\rightarrow} \sigma\left(\mathrm{t}_{1}, \ldots, \mathrm{t}_{\mathrm{n}}\right)
\end{gathered}
$$

The algebra $\mathrm{W}$ is the initial model of the $\sum$ if each operator of $\mathrm{W}$ is an operation of the syntactic construction of terms from syntactically compatible subterms.

\footnotetext{
${ }^{1}$ A grammar itself could be considered as a problem data. In these cases, an implicit formal specification, a signature and a semantic model, of a grammar is defined.

${ }^{2}$ Sets : A class of all finite, or infinite, countable sets.
} 
Two signatures could be related by a homomorphism.

Definition 2.7 ( $\Sigma$-homomorphism) Let A: $\Sigma \rightarrow$ Sets and B: $\Sigma \rightarrow$ Sets be two $\sum$-algebras, for $\sum: \Omega \rightarrow \mathrm{S}^{*} \mathrm{xS}$. Let $\mathrm{h}=\left(\mathrm{h}_{\mathrm{s}}\right)_{\mathrm{s} \in \mathrm{S}}$ be a family of functions over Sets with the following properties:
- for each $s \in \mathrm{S}$,
$\mathrm{h}_{\mathrm{s}}: \mathrm{A}(\mathrm{s}) \rightarrow \mathrm{B}(\mathrm{s})$

- for each $\sigma \in \Omega$ for which $\sigma: \mathrm{s}_{1} \times \ldots \times \mathrm{s}_{\mathrm{n}} \rightarrow \mathrm{s}$, h respects a functionality of $\sigma$, or

satisfying the diagram:

$$
\mathrm{h}_{\mathrm{s}}\left(\mathrm{A}(\sigma)\left(\mathrm{x}_{1}, \ldots, \mathrm{x}_{\mathrm{n}}\right)\right)=\mathrm{B}(\sigma)\left(\mathrm{h}_{\mathrm{s} 1}\left(\mathrm{x}_{1}\right), \ldots, \mathrm{h}_{\mathrm{sn}}\left(\mathrm{x}_{\mathrm{n}}\right)\right)
$$

$$
\begin{array}{cc}
\mathrm{A}(\sigma): \mathrm{A}\left(\mathrm{s}_{1}\right) \mathrm{x} \ldots \mathrm{x} & \mathrm{A}\left(\mathrm{s}_{\mathrm{n}}\right) \rightarrow \mathrm{A}(\mathrm{s}) \\
\downarrow \mathrm{h}_{\mathrm{s} 1} & \downarrow \mathrm{h}_{\mathrm{sn}} \\
\downarrow \mathrm{h}_{\mathrm{s}} \\
\mathrm{B}(\sigma): \mathrm{B}\left(\mathrm{s}_{1}\right) \mathrm{x} \ldots \mathrm{x}\left(\mathrm{B}\left(\mathrm{s}_{\mathrm{n}}\right) \rightarrow \mathrm{B}(\mathrm{s})\right.
\end{array}
$$

Then, $h$ is a homomorphism from $A$ to $B$, denoted $h: A \rightarrow B$.

Definition 2.8 ( $\sum$-interpretation) Let $\mathrm{A}=\mathrm{W}$ in the previous definition. Then, each $h_{s} W_{\Sigma, s} \rightarrow B(s)$ converts terms of the sort $s$ in values of $B(s)$ in a way that $\mathrm{h}_{\mathrm{s}}\left(\sigma\left(\mathrm{x}_{1}, \ldots, \mathrm{x}_{\mathrm{n}}\right)\right)=\mathrm{B}(\sigma)\left(\mathrm{h}_{\mathrm{s} 1}\left(\mathrm{x}_{1}\right), \ldots, \mathrm{h}_{\mathrm{sn}}\left(\mathrm{x}_{\mathrm{n}}\right)\right)$. That is, each term $\sigma\left(\mathrm{x}_{1}, \ldots, \mathrm{x}_{\mathrm{n}}\right)$ is converted to a value of $\mathrm{B}(\mathrm{s})$ in a structural way, combining by $\mathrm{B}(\sigma)$ the values $\mathrm{h}_{\mathrm{si}}\left(\mathrm{x}_{\mathrm{i}}\right)$, to which were converted all its subterms $\mathrm{t}_{\mathrm{i}}(1 \leq \mathrm{i} \leq \mathrm{n})$. This particular case of the homomorphism we call an interpretation of $\sum$-terms in an algebra B.

\section{CAD/CAPP MODEL}

\subsection{Syntactic description of models}

We will begin with a syntactic description of a CAD/CAPP system (in this case a "toy" system), defining a corresponded signature. At the first, we will define the signature of geometric transformations, $\Sigma_{\mathrm{G}}$, which specifies syntactically some operations in CAD, Figure 3.1.

$\mathrm{S}_{\mathrm{G}}=\{$ Structure, Point, Number, $\ldots\}$

$\Omega_{\mathrm{G}}=\{$ Move, Scaling, Subtract, Union, Intercept, Cylinder, Box, Sphere, $\mathrm{P}, \mathrm{N}\}$

$$
\sum_{G}=\left\{\begin{array}{llll}
\text { Move } & : \text { Structure } \times \text { Point } & \rightarrow & \text { Structure } \\
\text { Scaling } & : \text { Structure } \times \text { Number } & \rightarrow & \text { Structure } \\
\text { Subtract } & : \text { Structure } \times \text { Structure } & \rightarrow & \text { Structure } \\
\text { Union } & : \text { Structure } \times \text { Structure } & \rightarrow & \text { Structure } \\
\text { Intercept } & : \text { Structure } \times \text { Structure } & \rightarrow & \text { Structure } \\
\text { Cylinder } & : \text { Point } \times \text { Point } \times \text { Point } & \rightarrow & \text { Structure } \\
\text { Box } & : \text { Point } \times \text { Point } & \rightarrow & \text { Structure } \\
\text { Sphere } & : \text { Point } \times \text { Number } & \rightarrow & \text { Structure } \\
\mathrm{P} & : & \rightarrow & \text { Point } \\
\mathrm{N} & : & \rightarrow & \text { Number }
\end{array}\right.
$$

Figure 3.1 - Signature of a CAD system. 
Constants of the signature $\sum_{\mathrm{G}}$ are:

$$
\mathrm{C}_{\text {Number }}=\{\mathrm{N}\} \quad \mathrm{C}_{\text {Point }}=\{\} \quad \mathrm{C}_{\text {Structure }}=\{\}
$$

$\sum$-terms which could be obtained by this signature are:

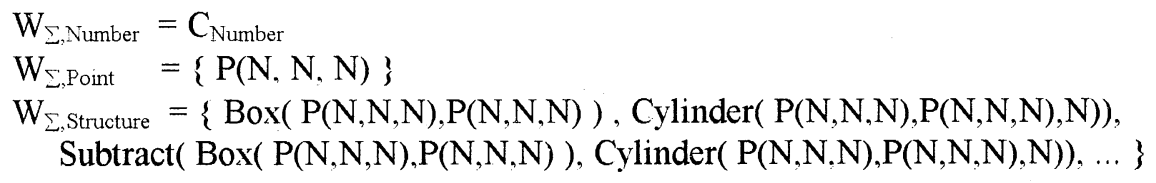

For example. the term "Subtract $(B o x(P(N, N, N), P(N, N, N))$, Cylinder $(P(N, N, N)$, $P(N, N, N), N)$ )" corresponds to the operation over objects, Figure 3.2. The syntax tree of the term is given on the Figure 3.3:

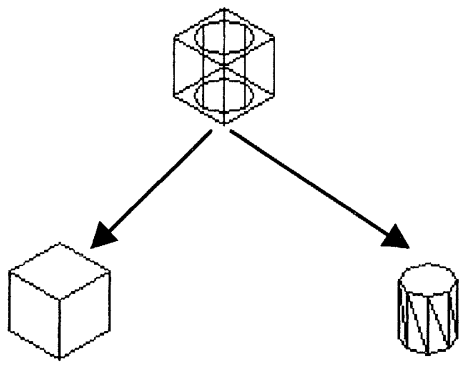

Figure 3.2 - Modelling of a box with a hole.

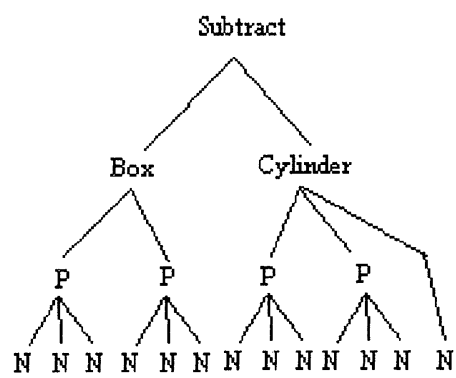

Figure 3.3 - Syntax tree of an abstract definition of the box with a hole.

\subsection{CAD and CAPP Model}

The signature describes only a syntactic nature of the CAD system. To attribute a meaning (semantics) to terms generated by the signature we have to define a semantic model. This model will be, in fact, a $\sum$-algebra. By the $\sum$-interpretation definition, a $C A D=\left(C A D_{S}, C A D_{\Omega}\right)$ semantic model could be defined as bellow. The model interprets the terms, generated by the signature (i.e., the model defines a meaning), on a (programming) language of implementation.

For a CAPP system we will use the same signature of the CAD system but with the different semantics (attributes).

CAD - Model:

$\begin{array}{ll}\mathrm{CAD}_{\mathrm{S}}(\text { Number }) & =\text { Tinteger } \\ \mathrm{CAD}_{\mathrm{S}}(\text { Structure }) & =\text { Tstructure } \\ \mathrm{CAD}_{\mathrm{S}}(\text { Point }) & =\text { Tpoint } \\ \mathrm{CAD}_{\Omega}(\text { Move }) & =\text { Move } \\ \mathrm{CAD}_{\Omega}(\text { Scaling }) & =\text { _Scaling } \\ \mathrm{CAD}_{\Omega}(\text { Subtract }) & =\text { _Subtract } \\ \mathrm{CAD}_{\Omega}(\text { Union }) & =\text { _Union } \\ \mathrm{CAD}_{\Omega}(\text { Cylinder }) & =\text { _Cylinder }\end{array}$

CAPP - Model:

$\begin{array}{ll}\operatorname{CAPP}_{S}(\text { Number }) & =\text { TInteger } \\ \operatorname{CAPP}_{S}(\text { Structure }) & =\text { TStructure } \\ \operatorname{CAPP}_{S}(\text { Point }) & =\text { TPoint } \\ \operatorname{CAPP}_{\Omega}(\text { Move }) & =\text { MoveFeature } \\ \operatorname{CAPP}_{\Omega}(\text { Scaling }) & =\text { ScaleFeature } \\ \operatorname{CAPP}_{\Omega}(\text { Subtract }) & =\text { SubProc } \\ \operatorname{CAPP}_{\Omega}(\text { Union }) & =\text { UnionProc } \\ \operatorname{CAPP}_{\Omega}(\text { Cylinder }) & =\text { FeatCylinder }\end{array}$



$\mathrm{CAD}_{\Omega}(\mathrm{Box})$
$=$ Box
$\mathrm{CAPP}_{\Omega}(\mathrm{Box})$
$=$ FeatureBox
$\mathrm{CAD}_{\Omega}$ ( Sphere )
$=-$ Sphere
$\mathrm{CAPP}_{\Omega}($ Sphere $)$
$=$ FeatureSphere
$\mathrm{CAD}_{\Omega}(\mathrm{P})$
$=-\mathrm{P}$
$\mathrm{CAPP}_{\Omega}(\mathrm{P})$
$=$ FeatureP
$\mathrm{CAD}_{\Omega}(\mathrm{N})$
$=-\mathrm{N}$
$\mathrm{CAPP}_{\Omega}(\mathrm{N})$
$=$ FeatureN

\subsection{CAD/CAPP Integration}

$\mathrm{CAD} / \mathrm{CAPP}$ integration is defined by a family of $\sum$-interpretation functions (recall $\Sigma$-homomorphism) which transform each term of the CAD algebra to a term of the CAPP algebra, $\mathrm{H}_{\mathrm{CAPP}}$ : $\mathrm{CAD} \rightarrow \mathrm{CAPP}$, in a structural way. In other words, each operation of the CAD system has its meaning (semantics) in the CAPP system.

As we need some kind of $\mathrm{CAPP}_{\Omega}{ }^{3}$ functions identification a corresponded knowledge base should be used as a tool for $\mathrm{CAPP}_{\Omega}$ functions identification. In order to facilitate the demonstration of CAD/CAPP formal specification we will use a pattern recognition knowledge base (which is probably the real base for CAD/CAPP integration), whose part is shown on the Figure 3.4.

Knowledge base for process planning $=$ \{
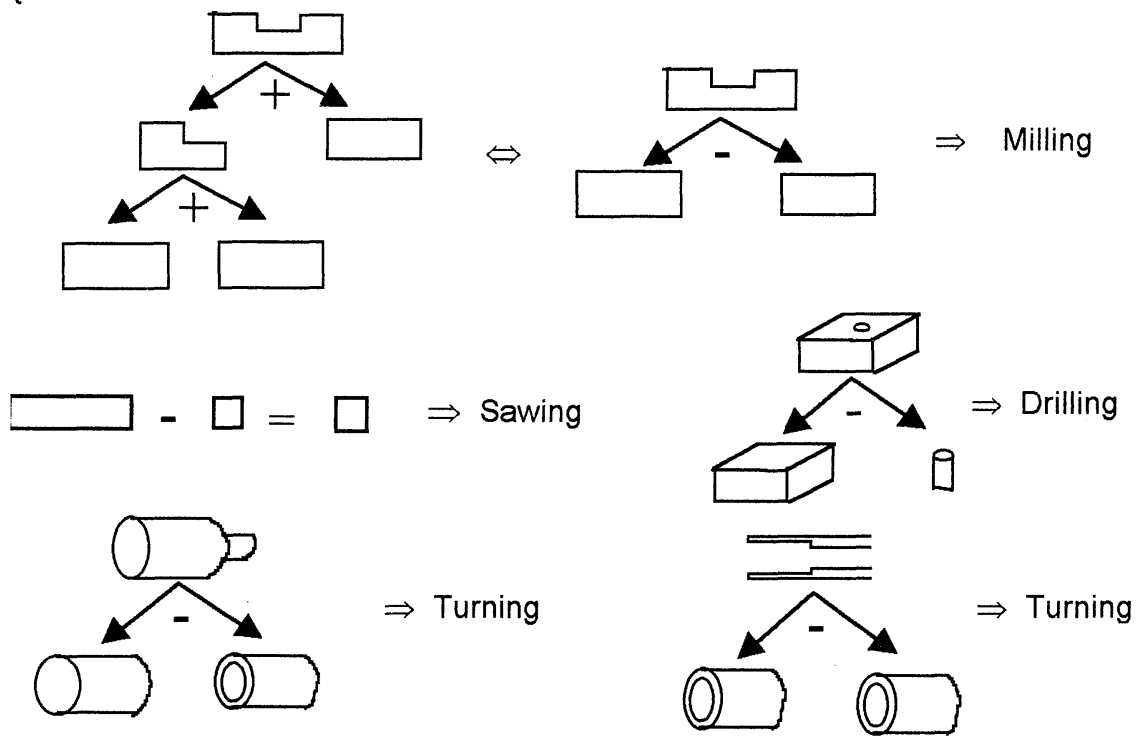

$\Rightarrow$ Turning

${ }^{3}$ Definition of processes coresponded to a $\mathrm{CAPP}_{\Omega}$ (milling, sawing, turning, ...) of a CAPP algebra, is very difficult because the relation between the geometric features and manufacturing processes is of the type n:m. Existing CAPP system use different types of resources, for example: data bases, knowledge bases, inference mechanisms, pattern recognition, machine learning, implemented through the variant and generative process planning concepts. 
Hardness(Initial) $>$ Hardness(Final) $\Rightarrow$ Heat treatment

Colour(Initial) $>$ Colour(Final) $\quad \Rightarrow$ Painting or Polishing or Zinc coating or ... ... $/ .$.

\}

Figure 3.4 - Knowledge base representation for process planning.

For example, a $\Sigma$-interpretation function ( $\sum$-homomorphism) for CAD/CAPP integration, for $\mathrm{CAD} / \mathrm{CAPP}$ example given, is defined as follows. If,

$$
h\left(\text { Subtract }_{\mathrm{CAD}}(\operatorname{Str} 1, \operatorname{Str} 2) \quad\right)=\text { Subtract }_{\mathrm{CAPP}}(\mathrm{h}(\operatorname{Str} 1), \mathrm{h}(\operatorname{Str} 2))
$$

then, after searching for corresponded patterns, we will have:

$$
\mathrm{A}_{\Omega}(\text { Subtract })=\text { Drilling }(\text { Str1, Str2 })
$$

\subsection{Grammar construction for CAD/CAPP integration}

Starting from the signature, we can define a grammar for our CAD system. In each production there is an additional string, which specifies a semantic operation associated to the recognition of the corresponded production.

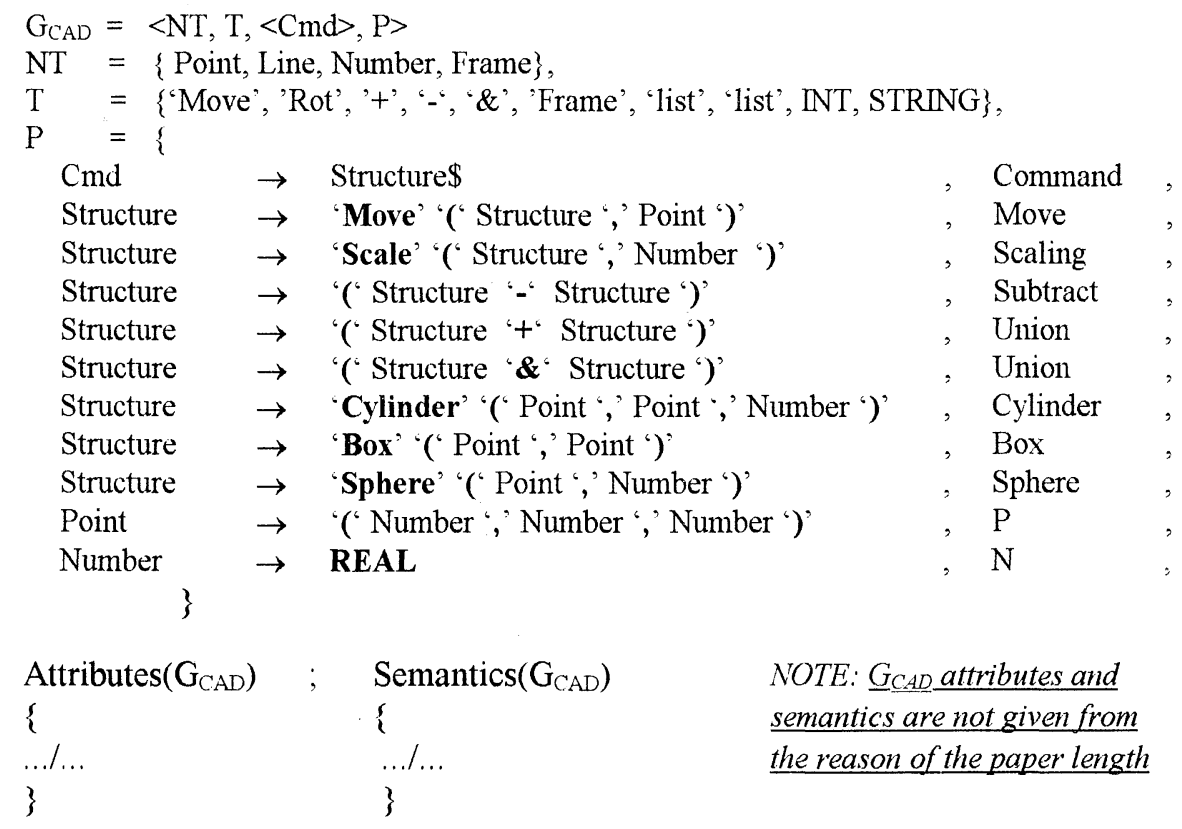

Figure 3.5 - Grammar for the CAD system

\section{PROCESS PLANNING: AN EXAMPLE}

Let the part given is represented on the Figure 4.1, with the features represented on the Figure 4.2. The objective is to obtain a manufacturing process sequence. 

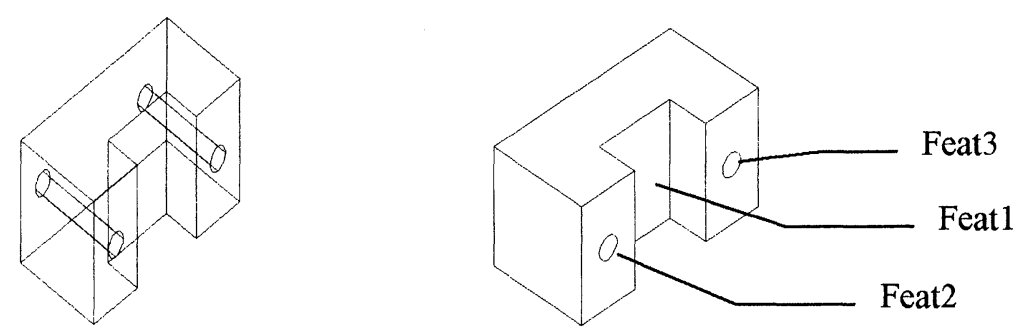

Figure 4.1 $\mathrm{CAD}$ model of the part. Figure 4.2 Features that belong to the part

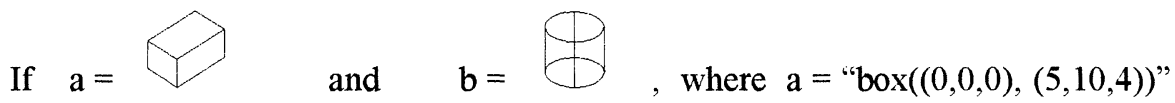
and $b=$ "cilynder $((0,0,0),(0,0,4), 1)$ " (the syntactic sub-trees of $a$ and $b$ will not be presented), then the solid (part) represented on the Figure 4.1 will be represented by the expression/phrase

$$
((((a-a)-a)-b)-b) \$
$$

which syntactic recognition results in a derivation tree ('parse tree'), Figure 4.3:

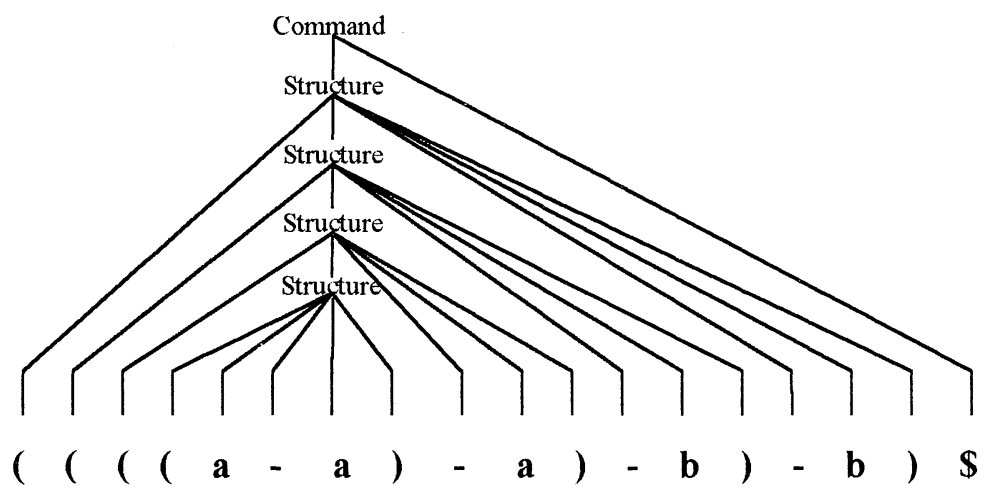

Figure 4.3 - Parse tree of a solid model string ( ( ( ( $a-a)-a)-b)-b) \$$.

Semantic operations, associated to the recognition of the syntactic expression, generate a data structure given on the Figure 4.4 (without the first term $(a-a)$ that represents "sawing"), which represents a kind of solid's semantic "figure".

Process planning consists of application of CAPP $\sum$-algebra operations to the variable TStructure, from left to the right, extracting manufacturing processes with the help of the pattern correspondence data base.

For example, on the first position we have the subtraction of two primitives corresponded to "blank" cutting (sawing process/operation). Applying the extraction process to all positions of the TStructure, we will obtain the sequence of processes which, basically, corresponds to the part's manufacturing process plan concept:

$<$ Sawing, Milling, Drilling, Drilling > 


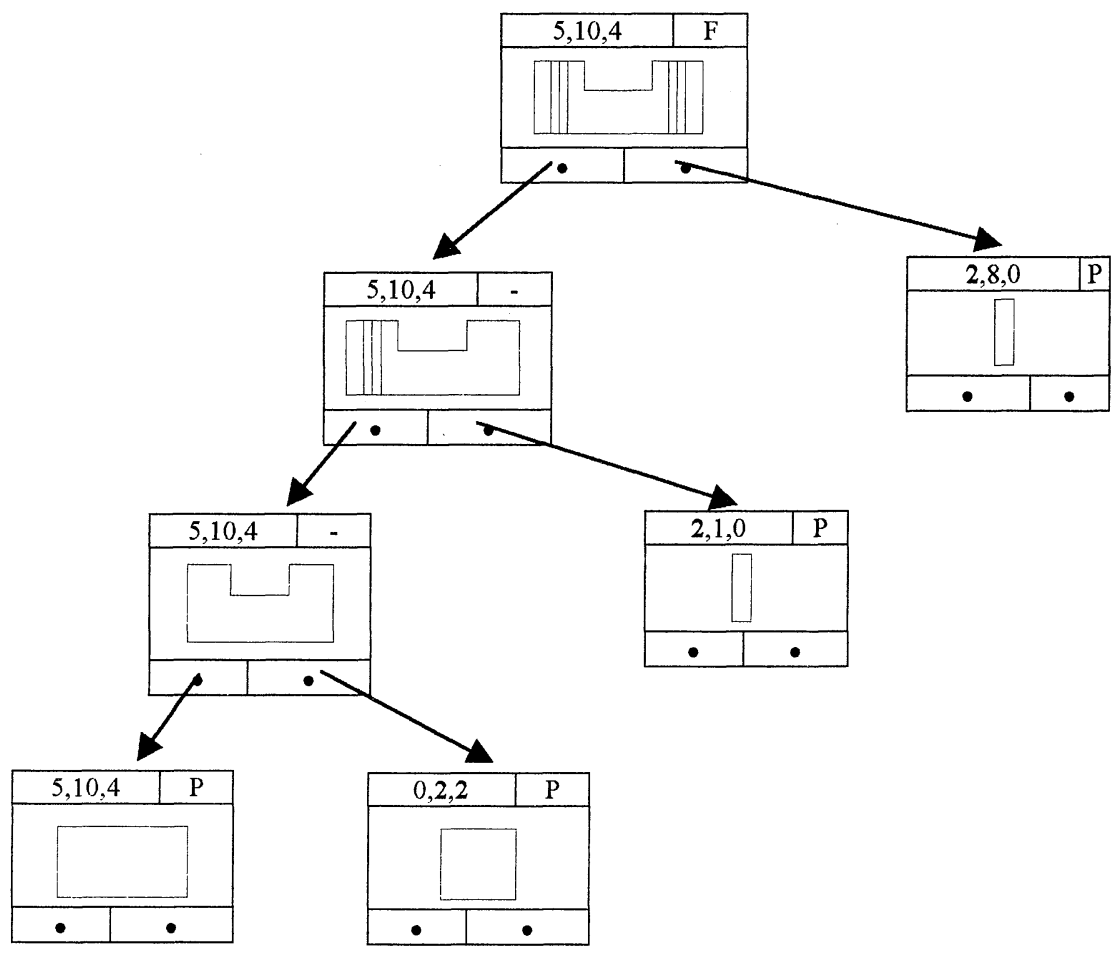

Figure 4.4 - Variable of the type TStructure after recognition

Processes identified, together with other complementary information contained in 'TStructure', on design and technological parameters (tolerance, surface quality, etc.) defined in the CAD model, as well as information about tools, machine-tools, part's material, etc., could be used by an algorithm which will calculate and determine operation elements, and their attributes, and, finally, manufacturing operations. To consider these parameters (tolerance, surface quality, tools, machine tools, part's material etc.) it is necessary an extension of the algebra presented.

\section{CONCLUSION}

The basic concept for a system specification and implementation oriented to formal models application were presented. The example presented shows how is possible to specify rigorously a problem without unnecessary details and its transduction to a concrete (programming) language. Even for a "toy" CAD/CAPP system given, it is impressive a condensity, or compactness, of its algebraic specification. The system's corresponded semantics and implementation emerge, almost intuitively. Deduction (automated generation), of the program is the method that guarantees correctness of its implementation and execution. 
We believe that this approach is an emerging issue in development of a manufacturing system (formal) theory. Naturally, if this observation is supposed to be true, the future work objectives are further more detailed applications of algebraic specification and semantic modelling to CAD/CAPP components and algorithms, and to other subsystems of a manufacturing system.

Regarding bibliography, two types of sources could be identified. Of the first type are sources belonging to pure mathematics, namely mathematical, formal logic, for example (Enderton; 1972), (Wagner; 1994.), important from the rigorous and abstract point of view independently of the application area. Of the second type are sources belonging to application areas (also rigorous in the formalism applied), i.e. to algebraic theory of engineering systems (almost exclusively to computer science), for example (Claßen et al.; 1993), (Hennessy; 1988), (Moll et al.; 1988.), (Oliveira J. N., 1993.), (Wechler; 1992.).

Unfortunately, we didn't find applications on manufacturing systems.

\section{REFERENCES}

Claßen I., Ehrig H., Wolz D. (1993) Algebraic Specification Techniques and Tools for Software Development, World Scientific.

Enderton B. E. (1972) A Mathematical Introduction to Logic, Academic Press. Hennessy M. (1988) Algebraic Theory of Processes, The MIT Press.

Moll R. N., Arbib M. A., Kfoury A. J. (1988) An Introduction to Formal Language Theory, Springer-Verlag.

Oliveira J. N. (1993) Especificação \& Semântica, Departamento de Informática Universidade do Minho.

Wagner E. G. (1994) Algebraic Semantics, in Abramsky S., Gabbay D. M.,

Maibaum T. C. E: Handbook of Logic in Computer Science - Vol. 3: Semantic structures, Clarendon Press, Oxford.

Wechler W. (1992) Universal Algebra for Computer Scientists, Springer-Verlag.

\section{BIOGRAPHIES}

João Almeida das Rosas received his Eng. Lic diploma from the University of Minho, Portugal, in the domain of Industrial Electronics Engineering. Eng. Rosas' current position is lecturer on the Escola Superior de Tecnologia e Gestão, Castelo Branco, Portugal. He is also a M.Sc. student at the University of Minho. His interests are CIM systems, design theory and process control and automation.

Dr. Goran D. Putnik received his M.Sci. and Dr.Sci. from the Belgrade University, both in domain of Intelligent Manufacturing Systems. His current position is assistant professor in the Department of Production and Systems Engineering, University of Minho, Portugal, for the subjects CAD/CAPP, Intelligent Systems for Manufacturing and Design Theory. His interests are machine learning and manufacturing system design and control theory and implementations. 